\title{
Changes in food intake patterns during 2000-2007 and 2008-2016 in the population-based Northern Sweden Diet Database
}

\author{
Ena Huseinovic ${ }^{1}$, Agneta Hörnell ${ }^{2}$, Ingegerd Johansson ${ }^{3}$, Anders Esberg $^{3}$, Bernt Lindahl ${ }^{4}$ and Anna Winkvist ${ }^{1,4^{*}}$ (D)
}

\begin{abstract}
Background: Food intake patterns provide a summary of dietary intake. Few studies have examined trends in food intake patterns over time in large, population-based studies. We examined food intake patterns and related sociodemographic and individual characteristics in the large Northern Sweden Diet Database during the two time windows 2000-2007 and 2008-2016.

Methods: In total, 100507 participants (51\% women) who had filled in a 64-item food frequency questionnaire and provided background and sociodemographic data between 2000 and 2016 were included. Food intake patterns were evaluated for women and men separately for the two time windows 2000-2007 and 2008-2016, respectively. Latent class analysis was used to identify distinct, latent clusters based on 40 food groups.

Results: Among both women and men, a greater proportion of participants were classified into food intake patterns characterized by high-fat spread and high-fat dairy during 2008-2016 compared to 2000-2007. In the earlier time window, these high-fat clusters were related to lower educational level and smoking. Simultaneously, the proportion of women and men classified into a cluster characterized by high intake of fruit, vegetables, and fibre decreased from the earlier to the later time window.

Conclusion: From a public health perspective, the increase in clusters with a high conditional mean for high-fat spread and high-fat dairy and decrease in clusters with a high conditional mean for fruit and vegetables, during the time period 2008-2016 compared to 2000-2007, is worrisome as it indicates a shift away from the recommended food habits. Subgroups of women and men with less healthy dietary patterns in the time window 2008-2016 with lower education, lower age, higher body mass index, lower levels of physical activity and more smoking were identified and future interventions may be targeted towards these groups.
\end{abstract}

Keywords: Food intake patterns, Dietary patterns, FFQ, NSDD, Diet, Time trends

\section{Background}

The Global Burden of Disease project annually provides data to quantify trends in health losses from hundreds of diseases and risk factors in 195 countries. The most recent update [1], found that in 2017 dietary risks were

\footnotetext{
* Correspondence: anna.winkvist@nutrition.gu.se

'Department of Internal Medicine and Clinical Nutrition, the Sahlgrenska Academy, University of Gothenburg, Box 459, SE-405 30 Gothenburg, Sweden

${ }^{4}$ Department of Public Health and Clinical Medicine, Section of Sustainable Health, Umeå University, Umeå, Sweden

Full list of author information is available at the end of the article
}

responsible for 11 million deaths and 255 million disability-adjusted life years (DALYs) globally. Dietary improvements could likely prevent one in every five deaths globally, and sub-optimal intakes of whole grains, fruits and sodium were reported to account for more than $50 \%$ of deaths and $66 \%$ of DALYs attributable to diet. Targeted interventions to improve dietary intake in populations is thus of high priority worldwide.

A large body of evidence identifies dietary habits of importance for maintaining good health [2-4]. Such healthy diets are rich in vegetables, pulses, fruit and

(c) The Author(s). 2019 Open Access This article is distributed under the terms of the Creative Commons Attribution 4.0 International License (http://creativecommons.org/licenses/by/4.0/), which permits unrestricted use, distribution, and 
berries, nuts and seeds, whole grain, fish and seafood, vegetable oil, and low-fat dairy. In addition to beneficial macro- and micronutrient content of these foods, they also provide several potentially bioactive components such as antioxidants, phenolic compounds, and phytoestrogens that may reduce the risk of non-communicable diseases. In contrast, diets characterized by high intake of red and processed meat, added sugars, fat, and salt have been associated with adverse health effects [2]. Understanding these dietary compounds and their specific framing within whole diets can help develop targeted interventions to improve health at the population level, not just by reducing consumption of unhealthy foods, but also by encouraging people to eat more of healthpromoting foods.

In line with these insights, the 5th edition of the Nordic Nutrition Recommendations (NNR) [2], as well as other nutrition recommendations (e.g [5]), that are used in planning diets for various populations have shifted focus from intake of single nutrients to the role of the whole diet, dietary patterns, and specific food groups. To decrease energy density, increase micronutrient density and improve carbohydrate and fat quality, NNR emphasize increased intake of fibre-rich plant foods, fish and seafood, nuts and seed, and vegetable oils and spreads, concurrent with decreased intake of sugar, high-fat dairy products, processed and red meat, and salt [2]. In practice, the advice provided based on the 5th edition of the NNR are similar to the advice based on previous editions.

Further work is now needed to identify pockets in the population with sub-optimal dietary patterns so that interventions can be targeted towards the correct population strata. Here, large population-based studies on dietary intake are needed that allow for comprehensive food pattern analyses. Unfortunately, few such population-based studies with detailed information on dietary intake and health exist, especially where diet has been monitored over time using the same methodology. Most often, Principal Component Analysis (PCA) has been used for generating dietary patterns in nutrition research. This is a "variablecentered" approach that identifies patterns among variables in the data set. A complementary approach is the person-centered, which identifies groups of people who have dietary behaviours in common. This may be more useful for later identifying at risk population groups. An example is here cluster analysis. A more recent technique is Latent Class Analysis (LCA), which has a number of statistical advantages over the more commonly used cluster and principal component analyses.

The Northern Sweden Diet Database (NSDD) [6], is a uniform database integrating self-reported questionnaire data on diet from several research projects in northern Sweden; the largest being the Västerbotten Intervention
Project (VIP). NSDD is one of the largest populationbased databases within a country in Europe, having monitored dietary intake among over 120000 individuals with the same methodology since 1985 . Using this database, we have previously reported food intake patterns among women and men in northern Sweden during the period 1992-2005 [7]. The aim of the current study was to evaluate associations between diet and health up to today and describe changes in dietary patterns over time using LCA. Hence, we examined food intake patterns and related sociodemographic and individual characteristics in the same cohort using data from the two time windows 2000-2007 and 2008-2016.

\section{Methods}

\section{Study population}

For the present analyses, only NSDD participants from VIP were included. The VIP study is an ongoing, population-based prospective study that runs in the county of Västerbotten in northern Sweden. The county has approximately 260000 inhabitants of which over 120000 live in the city of Umeå. Participants in VIP who had also provided dietary intake data to the NSDD were eligible for the present study. Since 1985, residents in the county have been invited by their local health center for a medical examination at 40,50 , and 60 years of age. Until 1996, 30-year olds were also invited, but for financial reasons today this only persists in some communities. Over the years, residents may have participated more than once. The average recruitment rate among available participants has been approximately $60 \%$. Only very limited evidence of selection bias in relation to income, age, and unemployment has been reported $[8,9]$. Also, no difference has been observed in cancer incidence in the VIP cohort versus in the general population of Västerbotten, indicating a truly population-based cohort [8].

VIP contained information on 114 642 observations (50.8\% women) between January 1st, 2000, to December 31,2016 . Observations were excluded from the present analyses if: food intake recording had $\geq 10 \%$ missing data and/or a missing portion indication; food intake recording included 84 items (see below); food intake level (calculated as the estimated total energy intake divided by basal metabolic rate) was extreme (i.e., highest and lowest $1 \%$ ); energy intake was extreme (lowest $1 \%$ and $>$ $5000 \mathrm{kcal}$ ); age $<29$ years or $>65$ years; height $<130$ or $>$ $210 \mathrm{~cm}$; weight $<35 \mathrm{~kg}$, or $\mathrm{BMI}<15$. This reduced the study sample to 100549 observations (Fig. 1). The data analyses in the present study were cross-sectional despite the longitudinal design of VIP.

Food intake patterns were analysed in two time windows for each gender: between 2000 and 2007 and 20082016, respectively. The cut point was chosen because it 


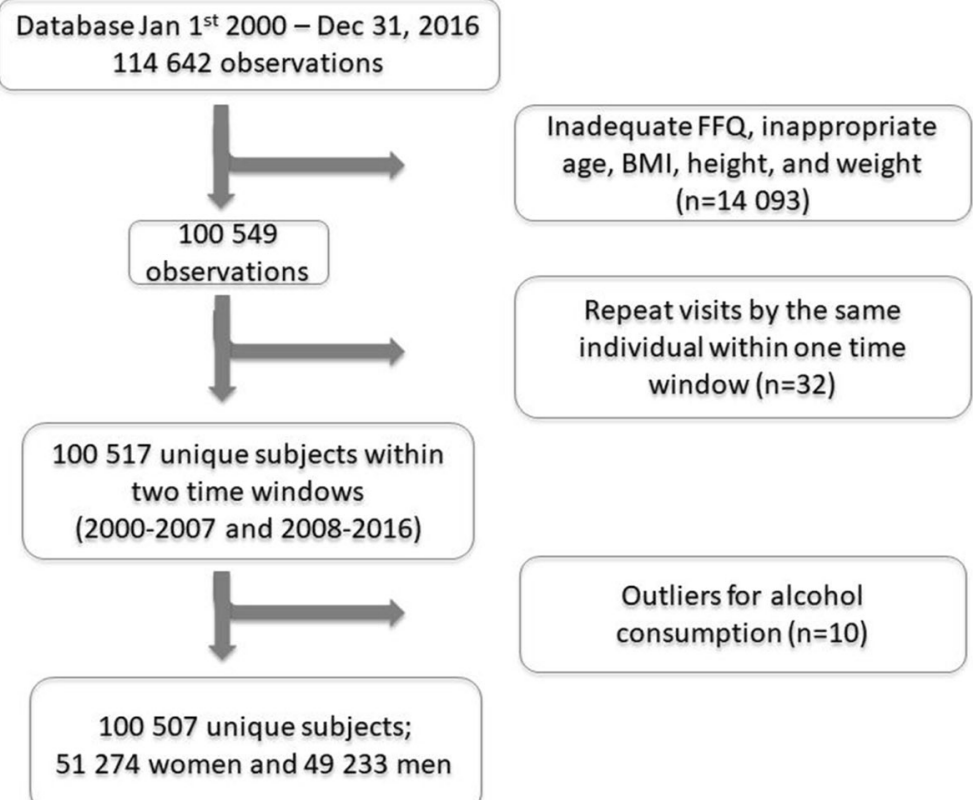

Fig. 1 Study flow chart. FFQ, Food Frequency Questionnaire; BMI, Body Mass Index

divided the overall period in two windows of equal size and also because around the years 2004-2007 a heated debate existed in media about low carbohydrate-high-fat diets $[10,11]$ that likely affected dietary intake trends [12] and possibly the cholesterol levels in the population [13]. Within each gender- and time specific window, repeat visits by the same individual were removed $(n=32)$. In addition, ten outliers for alcohol consumption were removed for women (four in 2000-2007 and six in 20082016). The final study sample thus consisted of 100507 unique subjects. The Research Ethics Committee at Umeå University approved the original study in 1984 (Dnr 2013/ 332/31) and the Regional Ethics Examination Board in Gothenburg approved the current study in 2017 (Dnr 276-17). Written informed consent was obtained from all participants.

\section{Diet measurements}

At the health visit, participants undergo an extensive health examination, including anthropometric measurements, blood pressure, serum lipid profiles, and an abbreviated oral glucose tolerance test. Also, participants answer an extensive questionnaire on lifestyle, health and life conditions, including a semi-quantitative Food Frequency Questionnaire (FFQ) that covers the preceding year [4]. Initially, the FFQ included 84 food items but from 1996 a shortened version of 64-66 food items was implemented. This reduction was achieved by deleting a few foods that were less common or by merging related food items. For the current analyses, only participants with the shorter FFQ versions were eligible.
In the FFQ, frequency of intake is reported on a 9level scale. Mealtime portion sizes are estimated with the support of four colour pictures of a plate containing increasing amounts of staple foods (potato/rice/pasta), main protein sources (meat/fish), and vegetables. For other foods, either gender- and age-specific portion sizes or fixed sizes (such as an apple or egg) are applied. Total estimated daily intake of energy (excluding energy from alcohol) and nutrients are calculated by weighting reported intake frequencies by food composition provided by the National Food Agency. Estimated intake of energy, nutrients, vitamins, and minerals has been validated against repeated 24-h dietary records [14, 15] and by serum biomarkers $[16,17]$ and found to have validity comparable to other FFQs used in cohort studies.

\section{Demographic, lifestyle and health variables}

Background characteristics were collected using detailed self-administered questionnaires including marital status (married/cohabiting and unmarried/other), education (secondary school and less, or academic education), and smoking (smoker, former smoker, and never smoker). Physical activity was measured using the Cambridge index of Physical Activity [18]. This is a validated index based on one question on occupational physical activity and one question on leisure time physical activity. Participants were categorized into inactive (sedentary job and no recreational activity), moderately inactive (sedentary job with $<0.5 \mathrm{~h}$ recreational activity per day, or standing job with no recreational activity), moderately active (sedentary job with $0.1-1 \mathrm{~h}$ recreational activity 
per day or standing job with $0.5 \mathrm{~h}$ recreational activity per day or physical job with no recreational activity), and active (sedentary job with $>1 \mathrm{~h}$ recreational activity per day or standing job with $>0.5 \mathrm{~h}$ recreational activity per day or physical job with at least some recreational activity or heavy manual job). Height was measured standing without shoes to the nearest $\mathrm{cm}$. Weight was measured in light clothing to the nearest $\mathrm{kg}$. BMI was calculated as weight $(\mathrm{kg})$ divided by the square of height $(\mathrm{m})$.

\section{Statistical analyses}

Food intake patterns were evaluated for the four different gender- and time specific data sets using LCA in Latent Gold 5.1 [19]. Similar to traditional cluster analysis, LCA groups individuals into mutually exclusive groups by minimizing the within-class variance and maximizing the between-class variance. However, instead of using the K-means algorithm and Euclidean distance to group individuals, LCA utilizes maximum likelihood algorithms and probabilities to identify distinct latent patterns based on a set of observed indicators (variables). Some of the advantages of LCA include the possibility to include indicators of different scale type in the model, provision of more formal criteria to determine the number of classes, 16 embedded random sets of start values, and no need to standardize indicators before analysis $[20,21]$. The method has previously been used to examine patterns of temporal eating $[22,23]$ and weight loss maintenance strategies [24].

For the present analysis, dietary data based on frequency of intake were used as indicators in the analysis. First, food items from the FFQ were grouped into 40 meaningful food groups according to nutrient content and/or culturally relevant culinary preferences (Table 1 ). To improve robustness of the analyses, frequency of intake was energy-adjusted by using frequency per 1000 $\mathrm{kcal}$. Thereafter, the food groups were entered as continuous indicators in Latent Gold 5.1 and conditional means were interpreted to determine distinguishing food groups within each class. The conditional mean reports the average number of times per day a respondent from latent class $\mathrm{k}$ consumes that food group. We initially explored the model fit of 1-7 latent classes by comparing the class solutions using the Bayesian information criteria (BIC), the LL statistics, class size, and pattern interpretability. After reaching the final model, differences in background characteristics and sociodemographics across the classes (or clusters as we refer to them in the remaining text) were examined using ANOVA and Chisquare test in SPSS version 25.0 (Armonk, NY: IBM Corp). For all analyses, $p<0.05$ was considered statistically significant. Values are presented as mean (SD) and proportions. The study adheres to the STROBE Nut reporting guidelines (Additional file 1).
Table 1 Food items included in the 40 food groups used in Latent Cluster Analyses

\begin{tabular}{|c|c|}
\hline Food group & Food items \\
\hline High-fat spreads & Butter and high-fat margarine ( $80 \%$ fat) \\
\hline Low-fat spreads & Low-fat margarine (40\% fat) \\
\hline Oil & $\begin{array}{l}\text { Vegetable oils in cooking and as salad } \\
\text { dressing }\end{array}$ \\
\hline Butter in cooking & Butter used in cooking \\
\hline Margarine in cooking & Margarine used in cooking \\
\hline Fruit & All fruits and berries \\
\hline High-fiber vegetables & Root vegetables, lettuce, cabbage, kale etc. \\
\hline Low-fiber vegetables & Tomato, cucumber \\
\hline Milk, 0.5\% & Milk, fermented milk $0.5 \%$ fat \\
\hline Milk, $1.5 \%$ & Milk, fermented milk $1.5 \%$ fat \\
\hline Milk, 3.0\% & Milk, fermented milk 3.0\% fat \\
\hline Cream & Cream, sour cream, crème fraiche \\
\hline High-fat cheese & Cheese, hard, $28 \%$ fat \\
\hline Low-fat cheese & Cheese, hard, $17 \%$ fat \\
\hline High-fiber cereals & $\begin{array}{l}\text { Oat-, graham- and rye porridge and } \\
\text { fiber-rich muesli }\end{array}$ \\
\hline Low-fiber cereals & Corn flakes etc. \\
\hline White bread & White bread, soft and hard \\
\hline High-fiber bread & High fiber bread, soft and hard \\
\hline Boiled potato & Boiled and mashed potato \\
\hline Fried potato & Fried potato and French fries \\
\hline Pasta and rice & Pasta, macaroni and rice \\
\hline Fish & High fat and lean fish, shellfish \\
\hline Red meat & Minced meat, stew, steak \\
\hline Bacon and sausage & Bacon and sausage \\
\hline Chicken & Chicken, hen \\
\hline Cold cuts & Meat, sausage and liverwurst on sandwich \\
\hline Pancakes and dumplings & Pancakes, dumplings \\
\hline Pulses & Beans, peas etc. \\
\hline Sweets & Candies, chocolate \\
\hline Sugar and jam & Sugar, marmalade, jam, honey \\
\hline Ice cream & Ice cream \\
\hline Cookies & Cookies, cakes \\
\hline Snacks & Chips, popcorn, peanuts \\
\hline Soda & Sodas \\
\hline Coffee & Coffee (boiled and filtered) \\
\hline Tea & Tea \\
\hline Beer & All types of beer \\
\hline Wine & Red and white wine \\
\hline Spirits & All types of spirits \\
\hline Fast food & Pizza and hamburger \\
\hline
\end{tabular}


As a complementary analysis of the emerging clusters, PCA and partial least squares (PLS) were run on the final data set to see if matching patterns were detected also with these data driven variable-centered approaches (Simca version 15.0, Umetrics, Sartorius Stedim Biotech, Umeå, Sweden). Input variables were the food groups created for the LCA, expressed as z-scores of frequency of intake per day and per $1000 \mathrm{kcal}$ to obtain standardization and energy adjustment. Factor selection was guided by $\mathrm{R}^{2}$ (the explained variation), $\mathrm{Q}^{2}$ (the predictive ability), Eigenvalues $>2.0$ and interpretability of the factors across samples. Analyses were carried out separately for women and men, and the two time windows, in line with the LCA.

\section{Results}

In total, 24360 women and 23107 men were included during 2000-2007 and 26914 women and 26126 men during 2008-2016 (Table 2). The mean age was approximately 50 years across all four data sets while BMI was lowest for women during 2000-2007 (mean (SD) 25.8 (4.6) $\mathrm{kg} / \mathrm{m}^{2}$ ), and highest for men during 2008-2016 $\left(27.2(4.1) \mathrm{kg} / \mathrm{m}^{2}\right)$. For both women and men, the majority of participants were married/cohabitating (range 78.5-81.5\%), had secondary school or less as highest educational level (range 55.7-75.5\%), and were nonsmokers (range 80.8-87.3\%).

\section{Latent classes of food intake patterns}

For all four data sets, model fit indices as well as pattern interpretability favored a four-cluster solution (see Additional file 2: Table S1, for model fit indices). Cluster labels were based on distinguishing features as shown by high conditional means of consuming the different food groups and numbered from largest to smallest group size (Figs. 2 and 3).

\section{Women during 2000-2007}

Among women during 2000-2007, the most common cluster was labeled High-fat dairy, white bread, sugar/ jam and cookies and comprised $39.7 \%$ of the participants in this sex and time window. This cluster had high conditional mean of consuming high-fat spread, full-fat milk, medium-fat milk, high-fat cheese, white bread, sugar/jam, and cookies. The second most common cluster was denoted Fruit, high-fiber bread and low-fat milk (35.2\% of the participants in this sex and time window) and was characterized by having high conditional mean of consuming fruit, low-fat milk, low-fat cheese, highfiber grains (i.e., bread and cereal), and low-fat spread. The third cluster, Bacon/sausage and fast food, comprised $23.5 \%$ of women and was characterized by high conditional mean of consuming bacon/sausage, fast food, snacks, red meat, cream, fried potato, pasta/rice, sweets, and soda. The fourth cluster, which comprised $1.6 \%$ of women and was labeled Pulses and tea, was characterized by high conditional mean of consuming especially pulses, but also tea and oil. This cluster and the Fruit, high-fiber bread and low-fat milk cluster both had high conditional mean of consuming several healthier foods as well as similar low conditional mean of consuming less healthy foods.

The High-fat dairy, white bread, sugar/jam and cookies cluster had the lowest proportion of women with academic education (29.0\%), and a low, although not the lowest, proportion of participants who reported to be physically active (24.0\%) (Table 3 ). In comparison, 54.8 and $36.6 \%$ of women in the Pulses and tea class reported to have academic education and to be physically active, respectively. Furthermore, fewer women in the Fruit, high-fiber bread and low-fat milk and Pulses and tea clusters reported to be current smokers (15.1 and 12.5\%, respectively) compared to the other two clusters (21.4 and $22.0 \%$, respectively), all $p<0.001$.

\section{Women during 2008-2016}

Among women during 2008-2016, the most common cluster was labeled High-fat spread and high-fat dairy, comprised the majority of women in this time window (70.9\%) and had high conditional mean of consuming high-fat spread, full-fat milk, medium-fat milk, and high-fat cheese. Women in the second most common cluster, Fruit, vegetables and oil (16.9\% of the participants), had high conditional mean of consuming fruit, vegetables, oil, high-fiber cereal, fish, chicken, pulses, tea, and wine. The third cluster, Sandwiches, comprised $10.4 \%$ of women and was characterized by high conditional mean of consuming low-fat spread, high-fiber bread, margarine in cooking, low-fat cheese, and cold cuts. Finally, $1.7 \%$ of women were categorized into the Soda and sweets cluster which was characterized by high conditional mean of consuming soda, sweets, sugar/jam, snacks, and fried potato.

Compared to the other three clusters, women in the Soda and sweets class were younger (47.2 years), had higher BMI $\left(27.2 \mathrm{~kg} / \mathrm{m}^{2}\right)$, and to a higher degree reported to be unmarried/other (28.1\%), physically inactive (18.3\%), and current smokers (23.9\%). In contrast, women in the Fruit, vegetables and oil cluster had the lowest proportion of participants who were physically inactive (9.9\%) and current smokers (8.7\%). In addition, this cluster had the lowest BMI $\left(26.0 \mathrm{~kg} / \mathrm{m}^{2}\right)$ and the highest proportion of women with academic education (53.8\%), all $p<0.001$ (Table 4).

\section{Men during 2000-2007}

Among men during 2000-2007, the most common cluster was denoted High-fat spread, high-fat dairy and 
Table 2 Background characteristics of participants in the Northern Sweden Diet Database during 2000-2007 and 2008-2016

\begin{tabular}{|c|c|c|c|c|}
\hline Background characteristic & $\begin{array}{l}\text { Women 2000-2007 } \\
(n=24360)\end{array}$ & $\begin{array}{l}\text { Women 2008-2016 } \\
(n=26914)\end{array}$ & $\begin{array}{l}\text { Men 2000-2007 } \\
(n=23 \text { 107) }\end{array}$ & $\begin{array}{l}\text { Men 2008-2016 } \\
(n=26126)\end{array}$ \\
\hline $\mathrm{Age}^{a}(\mathrm{yrs})$ & $50.1(8.0)$ & $50.3(8.2)$ & $50.1(8.0)$ & $50.2(8.2)$ \\
\hline Height $^{\mathrm{b}}(\mathrm{cm})$ & $165.1(6.0)$ & $165.5(6.2)$ & $178.7(6.5)$ & $179.3(6.7)$ \\
\hline Weight $^{\mathrm{b}}(\mathrm{kg})$ & $70.4(13.1)$ & $71.6(14.1)$ & $85.4(13.1)$ & $87.4(14.4)$ \\
\hline Body mass index $\left(\mathrm{kg} / \mathrm{m}^{2}\right)$ & $25.8(4.6)$ & $26.1(5.0)$ & $26.7(3.7)$ & $27.2(4.1)$ \\
\hline \multicolumn{5}{|l|}{ Marital status ${ }^{c}(\%)$} \\
\hline Married/cohabitating & 81.5 & 80.7 & 80.5 & 78.5 \\
\hline Unmarried/other & 18.5 & 19.3 & 19.5 & 21.5 \\
\hline \multicolumn{5}{|l|}{ Education (\%) } \\
\hline Secondary school or less & 65.4 & 55.7 & 75.5 & 69.5 \\
\hline Academic education & 34.6 & 44.3 & 24.5 & 30.5 \\
\hline \multicolumn{5}{|l|}{ Physical activity index ${ }^{d}(\%)$} \\
\hline Inactive & 14.9 & 13.4 & 16.4 & 15.3 \\
\hline Moderately inactive & 30.7 & 24.9 & 30.9 & 24.3 \\
\hline Moderately active & 27.6 & 27.6 & 29.3 & 30.0 \\
\hline Active & 26.8 & 34.1 & 23.4 & 30.4 \\
\hline \multicolumn{5}{|l|}{ Smoking (\%) } \\
\hline Smoker & 19.2 & 12.7 & 16.8 & 13.0 \\
\hline Former smoker & 32.5 & 32.7 & 33.9 & 28.6 \\
\hline Never smoker & 48.3 & 54.6 & 49.3 & 58.6 \\
\hline
\end{tabular}

Values are mean (SD) and proportions. ${ }^{a}$ Adjusted for year of study participation. ${ }^{\mathrm{b}}$ Adjusted for year of study participation and age. ${ }^{\mathrm{C}}$ In total, $125,59,111$, and 78 individuals are missing information on marital status, respectively. In total, 15932180,1101 , and 1900 individuals are missing information on physical activity, respectively

sugar/jam, and comprised $41.6 \%$ of the participants in this sex and time window. This cluster had high conditional mean of consuming high-fat spread, full-fat milk, medium-fat milk, and sugar/jam. The Sandwiches cluster (23.5\% of the participants) had high conditional mean of consuming low-fat spread, margarine in cooking, low-fat cheese, high-fiber bread, and cold cuts. The Fried potato and fast food cluster (18.4\% of the participants) was characterized by high conditional mean of consuming fried potato, fast food, pasta/rice, red meat, bacon/sausage, cream, sweets, snacks, soda, coffee, and beer. Finally, the Fruit, vegetables, oil and high-fiber cereals cluster (16.5\% of the participants) showed high conditional mean of fruit, vegetables, oil, low-fat milk, highfiber cereal, fish, pulses, tea, and wine.

The Fruit, vegetables, oil and high-fiber cereals cluster had higher age (52.3 years), lower proportion who were unmarried/other (15.7\%) and current smokers (11.5\%), and higher proportion who had academic education (39.6\%) and were physically active (28.3\%) compared to the other three clusters. In contrast, men in the Fried potato and fast food cluster were the youngest ( 45.7 years), while the High-fat spread, high-fat dairy and sugar/jam cluster had the highest proportion current smokers (18.9\%), all $p<0.001$, see Table 5.

\section{Men during 2008-2016}

Among men during 2008-2016, the most common cluster was labeled High-fat spread and high-fat dairy and comprised over half of the participants in this sex and time window (57.1\%). This cluster had high conditional mean of consuming high-fat spread, full-fat milk, and medium-fat milk. The second most common cluster, denoted Fast food, bacon/sausage and fried potato $(21.5 \%$ of participants), had high conditional mean of consuming fast food, bacon/sausage, fried potato, pasta/rice, sweets, snacks, soda, coffee, and beer. The two final groups comprised $10.7 \%$ each. Members of the Fruit, vegetables and oil cluster had high conditional mean of fruit, vegetables, oil, high-fiber cereal, fish, pulses, tea, and wine, while the cluster Sandwiches was characterized by low-fat spread, margarine in cooking, low-fat milk, low-fat cheese, white bread, high-fiber bread, and cold cuts.

The Fruit, vegetables and oil cluster had the highest proportion men who were married/cohabiting (81.9\%), had academic education (47.1\%), and were physically active (37.3\%) compared to the other three clusters. In contrast, men in the Fast food, bacon/sausage and fried potato class were the youngest (46.9 years) and had the highest proportion of current smokers (16.3\%), all $p<0.001$, see Table 6 . 


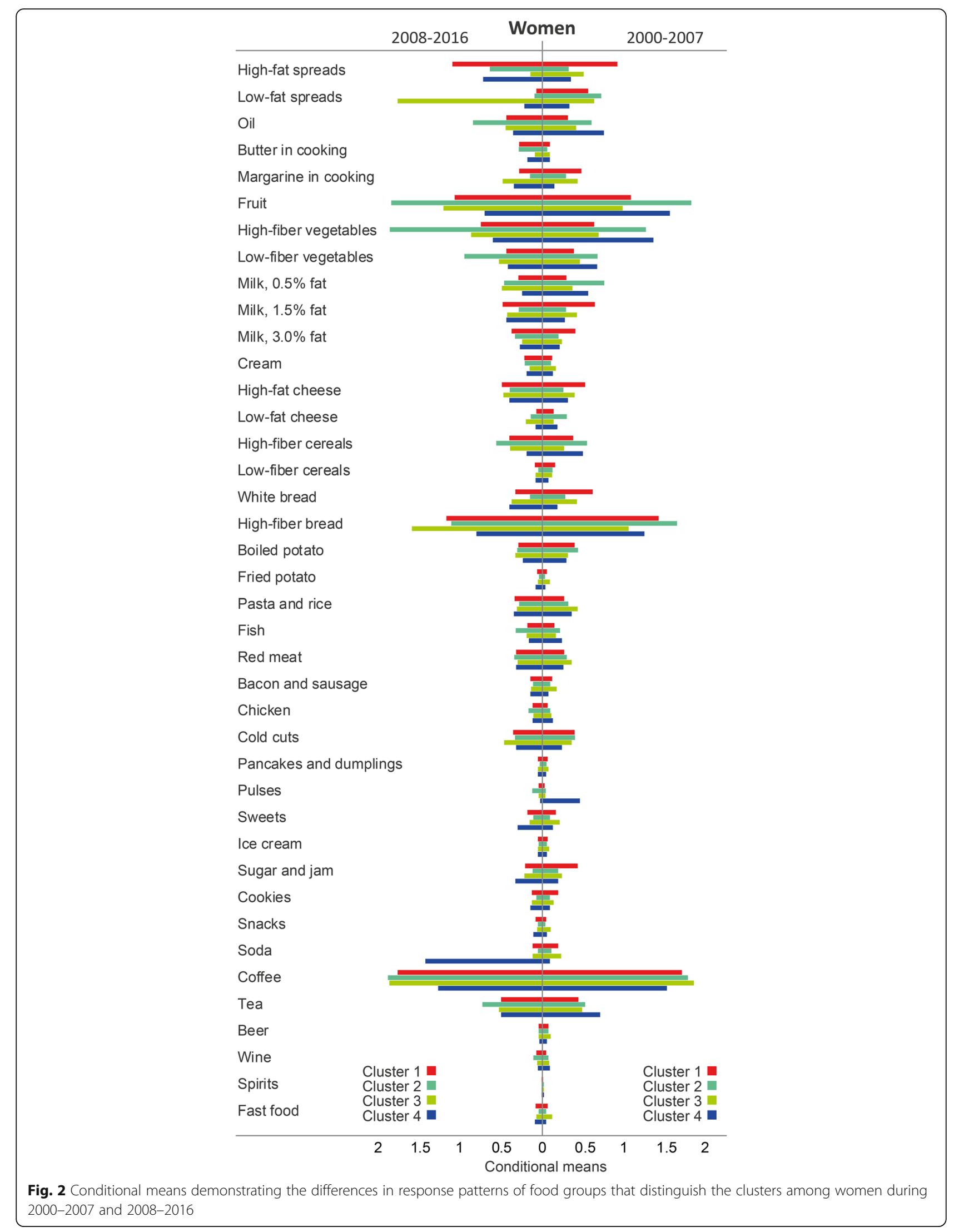




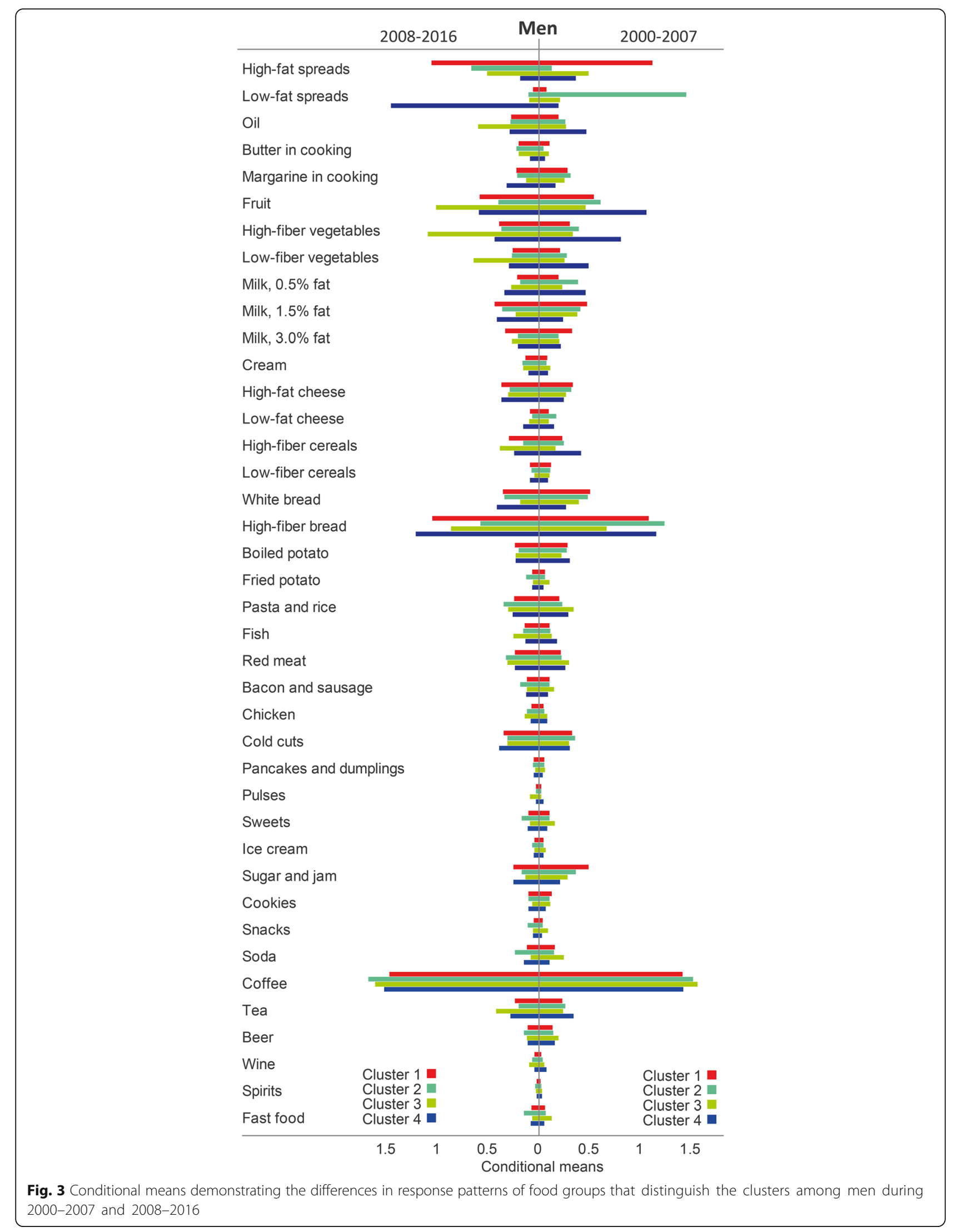


Table 3 Background data among food intake patterns during 2000-2007 for women in the Northern Sweden Diet Database ( $n=24$ 360)

\begin{tabular}{|c|c|c|c|c|c|}
\hline & Cluster 1 & Cluster 2 & Cluster 3 & Cluster 4 & $P$-value \\
\hline & $\begin{array}{l}\text { High-fat dairy, white bread, } \\
\text { sugar/jam and cookies }(n=9670)\end{array}$ & $\begin{array}{l}\text { Fruit, high-fiber bread and } \\
\text { low-fat milk }(n=8579)\end{array}$ & $\begin{array}{l}\text { Bacon/sausage and } \\
\text { fast food }(n=5726)\end{array}$ & $\begin{array}{l}\text { Pulses and tea } \\
(n=385)\end{array}$ & \\
\hline Age (yrs) & $50.4(8.0)$ & $52.7(7.5)$ & $45.8(7.1)$ & $50.0(8.0)$ & $<0.001$ \\
\hline Body weight (kg) & $69.7(13.1)$ & $71.4(12.9)$ & $70.3(13.2)$ & $69.4(13.4)$ & $<0.001$ \\
\hline Body mass index ${ }^{\mathrm{a}}\left(\mathrm{kg} / \mathrm{m}^{2}\right)^{\mathrm{a}}$ & $25.5(4.7)$ & $26.3(4.5)$ & $25.7(4.6)$ & $25.2(4.7)$ & $<0.001$ \\
\hline Marital status ${ }^{a}(\%)$ & & & & & $<0.001$ \\
\hline Married/cohabitating & 81.5 & 81.2 & 82.4 & 71.7 & \\
\hline Unmarried/other & 18.5 & 18.8 & 17.6 & 28.3 & \\
\hline Education (\%) & & & & & $<0.001$ \\
\hline Secondary school or less & 71.0 & 61.4 & 63.2 & 45.2 & \\
\hline Academic education & 29.0 & 38.6 & 36.8 & 54.8 & \\
\hline Physical activity index ${ }^{b}(\%)$ & & & & & $<0.001$ \\
\hline Inactive & 14.5 & 13.0 & 18.2 & 12.9 & \\
\hline Moderately inactive & 32.1 & 29.0 & 31.3 & 27.4 & \\
\hline Moderately active & 29.4 & 26.5 & 26.6 & 23.1 & \\
\hline Active & 24.0 & 31.5 & 23.9 & 36.6 & \\
\hline Smoking (\%) & & & & & $<0.001$ \\
\hline Smoker & 21.4 & 15.1 & 22.0 & 12.5 & \\
\hline Former smoker & 28.1 & 37.2 & 32.6 & 37.7 & \\
\hline Never smoker & 50.5 & 47.7 & 45.4 & 49.9 & \\
\hline
\end{tabular}

Values are mean (SD), and proportions. Continuous variables were analysed using ANOVA and categorical variables were analysed using Chi-square test. ${ }^{\text {In }}$ total, 125 women are missing information on marital status. ${ }^{b}$ In total, 1593 women are missing information on physical activity

\section{Results from complementary analyses with PCA and PLS}

To aid comparability with results from the LCA, score and loading plots were generated with LCA clusters included. For women 2000-2007, good separation was achieved for the first three LCA clusters in components 1 versus 2 (Additional file 3, panel A). The corresponding loadings plot revealed a spectrum on the horizontal axis from healthy (left) to unhealthy (right) foods and on the vertical axis from snacking components (bottom) to meals (top), where again the first three LCA clusters were distinctly separated (Additional file 3, panel C). When displaying components 1 versus 3 , the fourth cluster also was separated and this was largely driven by consumption of pulses (Additional file 3, panels B and $\mathrm{D}$, respectively).

For women 2008-2016, scores and loadings plots revealed clear separation for two of the LCA clusters, 1. High-fat spread and high-fat dairy; and 3. Sandwiches (Additional file 4, panels A and C). Cluster 2, Fruit, vegetables and oil, was separated in the loadings plot when comparing components 1 versus 2 (Additional file 4, panel C), and similar to women 2000-2007 spectra were visible on the horizontal axis from healthy (left) to unhealthy (right) foods and on the vertical axis from snacking components (bottom) to meals (top). Cluster 4, Soda and sweets, was separated in the loadings plot when comparing components 1 versus 3 and this was largely driven by consumption of soda (Additional file 4, panel D). Still, the scores plot was unable to identify good separation of clusters 2 and 4, even when inspecting components 1 versus 4 (Additional file 4, panel B).

For men 2000-2007, scores and loadings plots of components 1 versus 2 supported LCA results, showing good separation of all four LCA clusters (Additional file 5). Again, loadings spectra were visible on the horizontal axis from healthy (left) to unhealthy (right) foods and on the vertical axis from snacking components (bottom) to meals (top).

For men 2008-2016, scores plot showed separation of all LCA clusters except cluster 1, High-fat spread and high-fat dairy, and this cluster was not captured in further components either (Additional file 6, panel A). In corresponding loadings plot, all except cluster 4, Sandwiches, showed good separation (Additional file 6, panel B). Here, spectra were visible on the horizontal axis from meals (left) to snacking components (right) and on the vertical axis from unhealthy (bottom) to healthy (top) foods. When inspecting loadings of components 1 versus 3, also the fourth cluster was visible and this was largely driven by consumption of low-fat spread (Additional file 6, panel C). 
Table 4 Background data among food intake patterns during 2008-2016 for women in the Northern Sweden Diet Database $(n=26$ 914)

\begin{tabular}{|c|c|c|c|c|c|}
\hline & Cluster 1 & Cluster 2 & Cluster 3 & Cluster 4 & $P$-value \\
\hline & $\begin{array}{l}\text { High-fat spread and } \\
\text { high-fat dairy }(n=19093)\end{array}$ & $\begin{array}{l}\text { Fruit, vegetables and oil } \\
(n=4561)\end{array}$ & $\begin{array}{l}\text { Sandwiches } \\
(n=2 \text { 792) }\end{array}$ & $\begin{array}{l}\text { Soda and sweets } \\
(n=468)\end{array}$ & \\
\hline Age (yrs) & $49.5(8.1)$ & $52.8(7.7)$ & $52.1(8.1)$ & $47.2(7.7)$ & $<0.001$ \\
\hline Body weight (kg) & $71.6(14.2)$ & $70.8(13.1)$ & $72.6(14.6)$ & $74.2(16.4)$ & $<0.001$ \\
\hline Body mass index $\left(\mathrm{kg} / \mathrm{m}^{2}\right)$ & $26.1(5.0)$ & $26.0(4.7)$ & $26.6(5.2)$ & $27.2(5.8)$ & $<0.001$ \\
\hline Marital status ${ }^{a}(\%)$ & & & & & $<0.001$ \\
\hline Married/cohabitating & 81.3 & 78.6 & 81.4 & 71.9 & \\
\hline Unmarried/other & 18.7 & 21.4 & 18.6 & 28.1 & \\
\hline Education (\%) & & & & & $<0.001$ \\
\hline Secondary school or less & 56.4 & 46.2 & 64.7 & 66.0 & \\
\hline Academic education & 43.6 & 53.8 & 35.3 & 34.0 & \\
\hline Physical activity index ${ }^{b}(\%)$ & & & & & $<0.001$ \\
\hline Inactive & 14.2 & 9.9 & 13.3 & 18.3 & \\
\hline Moderately inactive & 25.1 & 21.8 & 27.6 & 27.8 & \\
\hline Moderately active & 28.4 & 23.8 & 28.2 & 27.1 & \\
\hline Active & 32.3 & 44.4 & 30.8 & 26.8 & \\
\hline Smoking (\%) & & & & & $<0.001$ \\
\hline Smoker & 13.0 & 8.7 & 15.0 & 23.9 & \\
\hline Former smoker & 31.3 & 38.6 & 33.7 & 27.4 & \\
\hline Never smoker & 55.6 & 52.7 & 51.3 & 48.7 & \\
\hline
\end{tabular}

Values are mean (SD) and proportions. Continuous variables were analysed using ANOVA and categorical variables were analysed using Chi-square test. ${ }^{\text {In }}$ total, 59 women are missing information on marital status. ${ }^{b}$ In total, 2180 women are missing information on physical activity

\section{Discussion}

We found that, when the two time windows were compared, both women and men in the latter time window appeared to move away from the Nordic recommendations on food habits [2]. A greater proportion of participants were classified into food intake patterns characterized by high-fat spread and high-fat dairy during 2008-2016 compared to 2000-2007. The increase was more than twice as large among women as among men $(+79 \%$ vs $+37 \%$, respectively). Perhaps this is an indication of a higher propensity for women to adopt new diets [25]. In the earlier time window, but not specifically in the later, these high-fat clusters were related to lower educational level and smoking. Simultaneously, the proportion of women and men classified into clusters characterized by high intake of fruit and vegetables decreased from the earlier to the later time window.

The VIP started in the mid-80s to decrease the prevalence of cardiovascular diseases in Västerbotten County, which then had the highest prevalence in Sweden [26]. The main components of the intervention were to decrease total fat intake, especially through decreased intake of saturated fat, and to increase the physical activity levels. Over the years, several studies have been performed on the growing dataset. One of these used a cross-sectional design to study 25-year time-trends in diet, cholesterol, and BMI between 1986 and 2010 [12]. The shift towards high-fat food patterns in the time-period 2008-2016 in the present study shows a continuation of a shift seen in that study [12]. After an initial decrease in intake of saturated and total fat between 1986 and 1992 among both men and women in the VIP-data, a sharp increase was seen from about 2004, followed by increasing levels of serum cholesterol after 2007 [13]. Although the cross-sectional design of that study made evaluation of causality between dietary fat intake and serum cholesterol levels impossible, the coinciding trends suggest a relationship. Interestingly, the increase in intake of saturated and total fat coincided with the introduction of positive media support for a low carbohydrate-high-fat diet [10] and criticism against official nutrition recommendations [11].

The common features of traditional Nordic dietary habits include a large proportion of milk and dairy products as well as moderate to high consumption of meat and fish, and a moderate intake of fruit and vegetables [2]. It is therefore not surprising that dairy intake was well represented in the largest clusters among women and men in both time segments. The question is whether the intake of saturated fats or the extra calories are of most concern in relation to cardiovascular health 
Table 5 Background data among food intake patterns during 2000-2007 for men in the Northern Sweden Diet Database ( $n=23$ 107)

\begin{tabular}{|c|c|c|c|c|c|}
\hline & Cluster 1 & Cluster 2 & Cluster 3 & Cluster 4 & $P$-value \\
\hline & $\begin{array}{l}\text { High-fat spread, high-fat dairy } \\
\text { and sugar/jam }(n=9604)\end{array}$ & $\begin{array}{l}\text { Sandwiches } \\
(n=5437)\end{array}$ & $\begin{array}{l}\text { Fried potato and } \\
\text { fast food }(n=4260)\end{array}$ & $\begin{array}{l}\text { Fruit, vegetables, oil and } \\
\text { high-fiber cereals }(n=3806)\end{array}$ & \\
\hline Age (yrs) & $50.7(8.0)$ & $50.9(7.9)$ & $45.7(6.9)$ & $52.3(7.7)$ & $<0.001$ \\
\hline Body weight (kg) & $84.1(12.7)$ & $86.6(13.5)$ & $86.0(13.2)$ & $86.2(13.2)$ & $<0.001$ \\
\hline Body mass index $\left(\mathrm{kg} / \mathrm{m}^{2}\right)$ & $26.3(3.6)$ & $27.1(3.8)$ & $26.8(3.8)$ & $27.0(3.7)$ & $<0.001$ \\
\hline Marital status ${ }^{a}(\%)$ & & & & & $<0.001$ \\
\hline Married/cohabitating & 79.0 & 82.4 & 78.2 & 84.3 & \\
\hline Unmarried/other & 21.0 & 17.6 & 21.8 & 15.7 & \\
\hline Education (\%) & & & & & $<0.001$ \\
\hline Secondary school or less & 80.6 & 79.4 & 72.9 & 60.4 & \\
\hline Academic education & 19.4 & 20.6 & 27.1 & 39.6 & \\
\hline Physical activity index ${ }^{b}(\%)$ & & & & & $<0.001$ \\
\hline Inactive & 14.9 & 17.4 & 18.0 & 17.1 & \\
\hline Moderately inactive & 31.0 & 31.5 & 31.8 & 28.9 & \\
\hline Moderately active & 31.4 & 28.0 & 29.3 & 25.7 & \\
\hline Active & 22.7 & 23.0 & 20.9 & 28.3 & \\
\hline Smoking (\%) & & & & & $<0.001$ \\
\hline Smoker & 18.9 & 16.0 & 18.1 & 11.5 & \\
\hline Former smoker & 32.6 & 36.6 & 29.7 & 38.0 & \\
\hline Never smoker & 48.6 & 47.4 & 52.2 & 50.5 & \\
\hline
\end{tabular}

Values are mean (SD) and proportions. Continuous variables were analysed using ANOVA and categorical variables were analysed using Chi-square test. ${ }^{\text {In }}$ total, 111 men are missing information on marital status. ${ }^{b}$ In total, 1101 men are missing information on physical activity

when considering high consumption of high-fat dairy and high-fat spreads from dairy. A recent review suggested that bioactive components associated with dairy lipids, especially high fat products, may be beneficial for cardio-metabolic health [27]. In a previous study in the same population, we found that the source of dairy fat, e.g., non-fermented or fermented milk, determined whether a high intake increased or decreased the risk for type 2 diabetes and myocardial infarction [28]. However, generally effect sizes were small.

The present study found relatively similar food patterns in the two time segments; however, the proportion of participants in each cluster showed large shifts. Still, changing food habits is not unique for the NSDD population. Swedish national data among adults from 1997 to 1998 [29] vs 2010-2011 [30] show changes in food habits over time, as well as differences between women and men. Here, intake of vegetables, pulses, fruit, and berries had increased in 2010-2011 compared to 19971998, especially among women. Likewise, intake of fish and seafood had increased while intake of potatoes had decreased among both women and men. The changes in food patterns in the present study do not exactly reflect the changes in these national studies. One reason for the discrepancies could be the different data collection methods. In both national studies, prospective food registrations were used, covering 7 days with 2027 participants in 1997-1998 [29], and 4 days with 2797 participants in 2010-2011 [30]. In contrast, the present study covered the habitual intake in the previous 12 months' food habits using a retrospective FFQ that has remained virtually unchanged since the mid-80's. Newer food stuffs may thus not be represented.

Using a FFQ with identical questions over an extended time period is a strength as well as a liability in longitudinal nutritional epidemiological studies. Favourably, it allows for correct evaluations of changes in intake of the food stuffs covered. However, if availability of different food items increases in grocery stores and restaurants, a more limited proportion of total food intake will be captured. A decrease in reported total energy intake has been noted in NSDD over a 10-year period when comparing intake data from two consecutive measurements for the same individuals (- $11.5 \%$ for women and$8.8 \%$ for men) concurrent with an increase in BMI (+4.0\% for women and $+3.8 \%$ for men) and slight increase in physical activity, indicating a need for further consideration [31].

In the present study, a decreased diversification in food patterns during the latter time period was visible, resulting in one dominating high-fat food pattern for both genders. This may reflect a decreased applicability 
Table 6 Background data among food intake patterns during 2008-2016 for men in the Northern Sweden Diet Database ( $n=26$ 126)

\begin{tabular}{|c|c|c|c|c|c|}
\hline & Cluster 1 & Cluster 2 & Cluster 3 & Cluster 4 & $P$-value \\
\hline & $\begin{array}{l}\text { High-fat spread and } \\
\text { high-fat dairy }(n=14905)\end{array}$ & $\begin{array}{l}\text { Fast food, bacon/sausage } \\
\text { and fried potato }(n=5629)\end{array}$ & $\begin{array}{l}\text { Fruit, vegetables and } \\
\text { oil }(n=2804)\end{array}$ & $\begin{array}{l}\text { Sandwiches } \\
(n=2788)\end{array}$ & \\
\hline Age (yrs) & $50.9(8.2)$ & $46.9(7.4)$ & $51.9(7.9)$ & $51.6(8.0)$ & $<0.001$ \\
\hline Body weight (kg) & $87.0(14.0)$ & $88.6(15.0)$ & $86.8(14.2)$ & $88.1(15.1)$ & $<0.001$ \\
\hline Body mass index $\left(\mathrm{kg} / \mathrm{m}^{2}\right)$ & $27.0(4.0)$ & $27.6(4.2)$ & $27.1(4.0)$ & $27.5(4.4)$ & $<0.001$ \\
\hline Marital status ${ }^{a}(\%)$ & & & & & $<0.001$ \\
\hline Married/cohabitating & 79.5 & 75.5 & 81.9 & 75.9 & \\
\hline Unmarried/other & 20.5 & 24.5 & 18.1 & 24.1 & \\
\hline Education (\%) & & & & & $<0.001$ \\
\hline Secondary school or less & 71.1 & 71.1 & 52.9 & 74.6 & \\
\hline Academic education & 28.9 & 28.9 & 47.1 & 25.4 & \\
\hline Physical activity index ${ }^{b}(\%)$ & & & & & $<0.001$ \\
\hline Inactive & 14.2 & 18.6 & 14.1 & 15.4 & \\
\hline Moderately inactive & 24.3 & 25.1 & 21.2 & 25.9 & \\
\hline Moderately active & 30.1 & 30.8 & 27.3 & 30.2 & \\
\hline Active & 31.4 & 25.5 & 37.3 & 28.5 & \\
\hline Smoking (\%) & & & & & $<0.001$ \\
\hline Smoker & 12.4 & 16.3 & 9.5 & 13.1 & \\
\hline Former smoker & 28.6 & 25.1 & 33.7 & 30.6 & \\
\hline Never smoker & 59.1 & 58.7 & 56.9 & 56.3 & \\
\hline
\end{tabular}

Values are mean (SD) and proportions. Continuous variables were analysed using ANOVA and categorical variables were analysed using Chi-square test. ${ }^{a}$ In total, 78 men are missing information on marital status. ${ }^{b}$ In total, 1900 men are missing information on physical activity

of the FFQ over time, especially for women where $70.9 \%$ belonged in the High-fat spread and high-fat dairy cluster. Although the results from the present analysis cannot be directly translated into trends in absolute intake, a decreased applicability of the FFQ may also partly explain the finding of a decreased proportion of participants in clusters characterized by intake of fruit and vegetables, which is contradictory to findings from the national studies $[29,30]$. Indeed, there has been large increases in availability of vegetables as well as vegetablebased alternatives to dairy in grocery stores in Sweden, and statistics from the Swedish Board of Agriculture indicate a $25 \%$ increase in purchases of vegetables in the Swedish population between 2000 and 2013 [32]. Such a large increase could be expected to be reflected also in the VIP-population. One explanation for the discrepancy could be that national statistics represent changes among the whole population while the present study only includes age groups who may be less inclined to change than younger segments of the population.

In the latest Swedish national dietary study on adults from 2010 to 11 [30], individuals with higher educational level reported higher consumption of fruit, berries, vegetables, cheese, and alcohol compared with individuals with lower educational level. This fits with the present study where groups with a high conditional mean of fruit, vegetables, and wine also had higher educational level in both time-periods for both gender. In both time periods for both sexes, clusters with high conditional mean of fruit and vegetables were associated with higher education, higher levels of physical activity and less smoking, indicating a healthier profile in this population strata. In the later time periods, clusters with high conditional mean of soda and sweets (women) and fast food, bacon/sausage and fried potato (men) were associated with younger age, higher BMI, less physical activity and more smoking, indicating a less healthy profile in this population strata.

The previous study on food patterns in NSDD [7] covered the period 1992-2005. It used cluster analysis and the number and combinations of food groups differed slightly from the present study. Similarities between the two studies include that clusters labelled Fruit and vegetables, and High fat, were identified in both studies and consisted of similar food groups. In the previous study, a cluster Coffee and sandwich was identified among women. In the current study, the cluster Sandwiches was identified in the later time period for both genders. Still, the previous study found a larger variation in frequency of intake among women than among men, resulting in four vs three distinct clusters, respectively. In the present study, both genders 
had four distinct clusters in both time periods; however the fourth cluster among women was based on one extreme food group and very small in both time periods, including less than $2 \%$ of the female population.

\section{Strengths and limitations of the study}

A major strength of the study is the large, populationbased sample of Swedish women and men including more than 100000 individuals between 2000 and 2016 . The large sample size permitted separate evaluations of women and men, and of two time periods so that trends over time may be captured. Food intake data were collected using the same methodology throughout the study period, thus permitting evaluations of changes in intake of food groups covered by the FFQ. As mentioned, this may however also imply a limitation in that more recent food stuffs are missed, leading to lower total intake and less variation in intake captured over time.

A novel feature of the study includes the use of LCA to identify latent subgroups within the study population with similar response patterns from the FFQ. Moreover, the study results were strengthened by complementary analyses using the data driven variable-centered methods PCA and PLS. Here, most clusters identified by LCA (except the very smallest) showed separation, and meaningful spectra ranging from healthy to unhealthy foods, and from full meals to snacking components, were visible in the loadings on food groups.

\section{Conclusion}

From a public health perspective, the increase in clusters with a high conditional mean for high-fat spread and high-fat dairy and decrease in clusters with a high conditional mean for fruit and vegetables, during the time period 2008-2016 compared to 2000-2007 is worrisome as it indicates a shift away from the recommended food habits. Subgroups of women and men with less healthy dietary patterns in the time window 2008-2016 with lower education, lower age, higher BMI, lower levels of physical activity and more smoking were identified and future interventions may be targeted towards these groups.

\section{Additional files}

Additional file 1: Adherence of the study according to STROBE-Nut. (DOC $84 \mathrm{~kb}$ )

Additional file 2: Table S1. Model fit indices for latent class models evaluated for the four data sets in the Northern Sweden Diet Database. (DOCX $13 \mathrm{~kb}$ )

Additional file 3: Principal component analysis model displaying separation for women during 2000-2007 related to belonging to the four clusters from Latent Class Analysis (Cluster 1, red. High-fat dairy, white bread, sugar/jam and cookies; Cluster 2, dark green. Fruit, low-fat milk and high-fiber bread; Cluster 3, light green. Bacon/sausage and fast food, and Cluster 4, blue. Pulses and tea). Panel A: Scores for components 1 versus 2. Panel B: Scores for components 1 versus 3. Further, loading plots corresponding to the score plots based on the 40 food groups responsible for the variation among the women. Location of the four clusters from Latent Class Analysis are indicated. Panel C: Loadings for components 1 vs 2. Panel D: Loadings for components 1 vs 3. (TIF 3129 kb)

Additional file 4: Principal component analysis model displaying separation for women during 2008-2016 related to belonging to the four classes/clusters from Latent Class Analysis (Cluster1, red. High-fat spread and high-fat dairy; Cluster 2, dark green. Fruit, vegetables and oil; Cluster 3, light green. Sandwiches, and Cluster 4, blue. Soda and sweets). Panel A: Scores for components 1 versus 2. Panel B: Scores for components 1 versus 4. Further, loading plots corresponding to the score plots based on the 40 food groups responsible for the variation among the women. Location of the four classes/clusters from Latent Class Analysis are indicated. Panel C: Loadings for components 1 versus 2. Panel D: Loadings for components 1 versus 3. (TIF $5427 \mathrm{~kb}$ )

Additional file 5: Principal component analysis model displaying separation for men during 2000-2007 related to belonging to the four clusters from Latent Class Analysis (Cluster 1, red. High-fat spread, high-fat dairy and sugar/jam; Cluster 2, dark green. Sandwiches; Cluster 3, light green. Fried potato and fast food, and Cluster 4, blue. Fruit, vegetables, oil and high-fiber cereals). Panel A: Scores for components 1 versus 2. Further, loading plots corresponding to the score plot based on the 40 food groups responsible for the variation among the men. Location of the four clusters from Latent Class Analysis are indicated. Panel B: Loadings for components 1 versus 2. (TIF 4014 kb)

Additional file 6: Principal component analysis model displaying separation for men during 2008-2016 related to belonging to the four clusters from Latent Class Analysis (Cluster 1, red. High-fat spread and high-fat dairy; Cluster 2, dark green. Fast food, bacon/sausage and fried potato; Cluster 3, light green. Fruit, vegetables and oil, and Cluster 4, blue. Sandwiches). Panel A. Scores for components 1 versus 2. Further, loading plots corresponding to the score plots based on the 40 food groups responsible for the variation among the men. Location of the four clusters from Latent Class Analysis are indicated. Panel B: Loadings for components 1 versus 2. Panel C: Loadings for components 1 versus 3. (TIF 5241 kb)

\section{Abbreviations}

BIC: The Bayesian Information Criteria; BMI: Body mass index $\left(\mathrm{kg} / \mathrm{m}^{2}\right)$; DALYs: Disability-adjusted life years; FFQ: Food Frequency Questionnaire; LCA: Latent Class Analysis; NSDD: The Northern Sweden Diet Database; PCA: Principal components analysis; PLS: Partial least squares; VIP: Västerbotten Intervention Project

\section{Acknowledgements}

The authors want to acknowledge professor Göran Hallmans for support in forming NSDD, teams at Västerbotten County Council for collecting data and organizing VIP, and the personnel at the Department of Biobank Research, Umeå University, Sweden for data maintenance and administrative support. We also thank Jay Magidson for assisting with interpretation of the Latent Class Analyses.

\section{Authors' contributions}

AW and $\mathrm{EH}$ designed the project; $\mathrm{EH}$ analyzed the data; $\mathrm{EH}$ drafted the manuscript with support from $\mathrm{AW}$ and $\mathrm{AH} ; \mathrm{AW}, \mathrm{IJ}$ and $\mathrm{AE}$ contributed with analyses using PCA and PLS, and BL contributed with interpretation of medical aspects. All authors participated in manuscript revision and all approved the final manuscript.

\section{Funding}

The Northern Sweden Diet Database was supported by the Swedish Research Council for Health, Working Life and Welfare (FORTE) and Vetenskapsrådet (the Swedish Research Council). The present study was supported by the Swedish Research Council (2016-01216).

\section{Availability of data and materials}

The datasets generated and analyzed during the current study are not publicly available due to Swedish legislations but are available from the 
Department of Biobank Research, Umeå University, on reasonable request and with appropriate ethical approval and approval of the local expert group. Detailed information is available on the website (https://www.umu.se/en/ biobank-research-unit/).

\section{Ethics approval and consent to participate}

The study protocol and data handling procedures were approved by the Regional Ethical Review Board of Northern Sweden, Umeå (Dnr 2013/332/31) and the Regional Ethics Examination Board in Gothenburg approved the current study in 2017 (Dnr 276-17). All study participants provided written informed consent, and the study was conducted in accordance with the Declaration of Helsinki.

\section{Consent for publication}

Not applicable.

\section{Competing interests}

The authors declare that they have no competing interests.

\section{Author details}

${ }^{1}$ Department of Internal Medicine and Clinical Nutrition, the Sahlgrenska Academy, University of Gothenburg, Box 459, SE-405 30 Gothenburg, Sweden. ${ }^{2}$ Department of Food and Nutrition, Umeå University, Umeå, Sweden. ${ }^{3}$ Department of Odontology, Umeå University, Umeå, Sweden. ${ }^{4}$ Department of Public Health and Clinical Medicine, Section of Sustainable Health, Umeå University, Umeå, Sweden.

\section{Received: 20 February 2019 Accepted: 4 July 2019}

\section{Published online: 12 July 2019}

\section{References}

1. GBD 2017 Diet Collaborators. Health effects of dietary risks in 195 countries, 1990-2017: a systematic analysis for the Global Burden of Disease Study 2017. Lancet. 2019;393:1958.

2. Nordic Council of Ministers 2014. Nordic Nutrition Recommendations 2012: Integrating nutrition and physical activity. 5th edition. Copenhagen: Norden; 2014.

3. Martinez-Gonzalez MA, Bes-Rastrollo M, Serra-Majem L, Lairon D, Estruch R, Trichopoulou A. Mediterranean food pattern and the primary prevention of chronic disease: recent developments. Nutr Rev. 2009;67(Suppl 1):S111-6.

4. Shen J, Wilmot KA, Ghasemzadeh N, Molloy DL, Burkman G, Mekonnen G, et al. Mediterranean dietary patterns and cardiovascular health. Annu Rev Nutr. 2015;35:425-49.

5. WHO. Healthy diet. Fact sheet N 394, updated Aug 2018.

6. Swedish National Data Service SND. Northern Sweden Diet Database (NSDD): Umeå University, Department of Biobank Research 2018 [updated 2018; cited 2018 Dec 20]. Available from: https:/snd.gu.se/en/catalogue/study/ ext0131.

7. Winkvist A, Hörnell A, Hallmans G, Lindahl B, Weinehall L, Johansson I. More distinct food intake patterns among women than men in northern Sweden: a population-based survey. Nutr J. 2009;8:12.

8. Pukkala E, Andersen A, Berglund G, Gislefoss R, Gudnason V, Hallmans G, et al. Nordic biological specimen banks as basis for studies of cancer causes and control--more than 2 million sample donors, 25 million person years and 100,000 prospective cancers. Acta Oncol. 2007;46:3.

9. Weinehall L, Hallgren CG, Westman G, Janlert U, Wall S. Reduction of selection bias in primary prevention of cardiovascular disease through involvement of primary health care. Scand J Prim Health. 1998;16(3).

10. Gunnarsson A, Elam M. Food fight! The Swedish low-carb/high fat (LCHF) movement and the turning of science popularisation against the scientists. Sci Cult. 2012;21(3).

11. Mann J, Nye ER. Fad diets in Sweden, of all places. Lancet. 2009;374(9692):767-9.

12. Johansson I, Nilsson LM, Stegmayr B, Boman K, Hallmans G, Winkvist A. Associations among 25-year trends in diet, cholesterol and BMI from 140,000 observations in men and women in northern Sweden. Nutr J. 2012;11:40.

13. $\mathrm{Ng} \mathrm{N}$, Johnson $\mathrm{O}$, Lindahl B, Norberg M. A reversal of decreasing trends in population cholesterol levels in Vasterbotten County, Sweden. Glob Health Action. 2012;5:10367.
14. Johansson I, Hallmans G, Wikman A, Biessy C, Riboli E, Kaaks R. Validation and calibration of food-frequency questionnaire measurements in the northern Sweden health and disease cohort. Public Health Nutr. 2002;5(3).

15. Klingberg S, Winkvist A, Hallmans G, Johansson I. Evaluation of plant sterol intake estimated with the northern Sweden FFQ. Public Health Nutr. 2013;16(3).

16. Wennberg M, Vessby $B$, Johansson I. Evaluation of relative intake of fatty acids according to the northern Sweden FFQ with fatty acid levels in erythrocyte membranes as biomarkers. Public Health Nutr. 2009;12(9).

17. Johansson I, Van Guelpen B, Hultdin J, Johansson M, Hallmans G, Stattin P. Validity of food frequency questionnaire estimated intakes of folate and other B vitamins in a region without folic acid fortification. Eur J Clin Nutr. 2010;64(8).

18. Peters T, Brage S, Westgate K, Franks PW, Gradmark A, Tormo Diaz MJ, et al. Validity of a short questionnaire to assess physical activity in 10 European countries. Eur J Epidemiol. 2012;27:1.

19. Vermunt JK, Magidson J. Latent class cluster analysis. In: Hagenaars JA, McCutcheon AL, editors. Applied latent class analysis. Cambridge: Cambridge University Press; 2002. p. 89-106

20. Vermunt JK, Magidson J. Upgrade manual for latent GOLD 5.1 Belmont Massachusetts: Statistical Innovations Inc; 2016.

21. Vermunt JK, Magidson J. Latent GOLD 4.0 User's Guide. Belmont, Massachusetts: Statistical Innovations Inc; 2005.

22. Leech RM, Worsley A, Timperio A, McNaughton SA. Temporal eating patterns: a latent class analysis approach. Int J Behav Nutr Phys Act. 2017;14:1.

23. Leech RM, Timperio A, Worsley A, McNaughton SA. Eating patterns of Australian adults: associations with blood pressure and hypertension prevalence. Eur J Clin Nutr. 2018. https://doi.org/10.1007/s00394-018-1741-y.

24. Ogden LG, Stroebele N, Wyatt HR, Catenacci VA, Peters JC, Stuht J, et al. Cluster analysis of the national weight control registry to identify distinct subgroups maintaining successful weight loss. Obesity. 2012;20(10).

25. Bärebring L, Winkvist A, Augustin H. Sociodemographic factors associated with reported attempts at weight loss and specific dietary regimens in Sweden: the SWEDIET-2017 study. PLoS One. 2018;13:5.

26. Weinehall L, Hellsten G, Boman K, Hallmans G. Prevention of cardiovascular disease in Sweden: the Norsjö community intervention programme--motives, methods and intervention components. Scand J Public Health Suppl. 2001;56: 13-20.

27. Lordan R, Tsoupras A, Mitra B, Zabetakis I. Dairy fats and cardiovascular disease: do we really need to be concerned? Foods. 2018;7(3). https://doi. org/10.3390/foods7030029.

28. Johansson I, Esberg A, Nilsson LM, Jansson JH, Wennberg P, Winkvist A. Dairy product intake and cardiometabolic diseases in northern Sweden: a 33-year prospective cohort study. Nutrients. 2019;11(2). https://doi.org/10.33 90/nu11020284.

29. Becker W, Pearson M. Riksmaten 1997-1998. Kostvanor och näringsintag i Sverige-Metod-och resultatanalys. Uppsala: Livsmedelsverket. [Diet habits and nutrient intake in Sweden-methods and results].

30. Swedish National Food Administration. National Food Consumption Survey 2010-11. Uppsala, Sweden, 2012.

31. Winkvist A, Klingberg S, Nilsson LM, Wennberg M, Renstrom F, Hallmans G, et al. Longitudinal 10-year changes in dietary intake and associations with cardio-metabolic risk factors in the northern Sweden health and disease study. Nutr J. 2017;16(1).

32. Swedish Board of Agriculture. Livsmedelskonsumtionen i siffror - Hur har konsumtionen utvecklats de senaste femtio åren och varför? Rapport 2015: 15. [Food consumption in numbers-how has consumption developed during the last fifty years and why? Report].

\section{Publisher's Note}

Springer Nature remains neutral with regard to jurisdictional claims in published maps and institutional affiliations. 